\title{
Cardiac Resynchronization and Defibrillator Therapy (CRT-D) or CRT Alone (CRT-P) in patients with dilated cardiomyopathy and heart failure without late gadolinium enhancement (LGE) cardiac magnetic resonance imaging (CMRI) high-risk markers - CRT-REALITY study - Study design and rationale
}

\author{
Milos Taborsky ${ }^{\mathrm{a}}$, Tomas Skala ${ }^{\mathrm{a}}$, Renata Aiglova ${ }^{\mathrm{a}}$, Marian Fedorco ${ }^{\mathrm{a}}$, Josef Kautzner ${ }^{\mathrm{b}}$, Tomas Jandikc, Vlastimil Vancurac, \\ Ales Linhart ${ }^{\mathrm{d}}$, Martin Valek ${ }^{\mathrm{d}}$, Miloslav Novak ${ }^{\mathrm{e}}$, Petr Kala ${ }^{\mathrm{f}}$, Rostislav Polasek ${ }^{\mathrm{g}}$, Tomas Roubicek ${ }^{\mathrm{g}}$, Alexandr Schee ${ }^{\mathrm{h}}$, \\ Gerhard Hindricksi, Nikolaos Dagres', Robert Hatalaj, Jiri Jarkovskyk
}

Background. Primary preventive implantation of implantable defibrillator (ICD) is according to current guidelines indicated in patients with heart failure NYHA (New York Heart Association) class II/III and LVEF $<35 \%$. Thanks to advances in heart failure pharmacotherapy, a decrease in mortality could render a benefit of ICD insufficient to justify its implantation in some patients.

Methods. Study design: multicenter, prospective, randomized, controlled trial evaluating the benefit of implantation of Cardiac Resynchronization and Defibrillator Therapy (CRT-D) or CRT Alone (CRT-P) in non-ischemic patients with reduced left ventricle ejection fraction (LVEF) and optimal pharmacotherapy without significant mid-wall myocardial fibrosis detected by cardiac magnetic resonance (CMR). The primary end-point: Re-hospitalization for heart failure, ventricular tachycardia, major adverse cardiac events (MACE). The secondary end-points: Sudden cardiac death, cardiovascular death, resuscitated cardiac arrest or sustained ventricular tachycardia, device-related complications, and change in quality of life. Course of the study: After a pharmacotherapy is optimized and significant mid-wall myocardial fibrosis excluded, patients will be randomized 1:1 to CRT-P or CRT-D implantation.

Discussion. If our hypothesis is confirmed, this could provide evidence for the management of these patients with a significant impact on common daily praxis and health care expenditures.

Trial registration. ClinicalTrials.gov, NCT04139460

Key words: non-ischemic cardiomyopathy, heart failure, implantable cardioverter-defibrillator, cardiac resynchronization therapy, magnetic resonance imaging, late gadolinium enhancement, randomized controlled trial

Received: December 15, 2020; Revised: December 15, 2020; Accepted: February 5, 2021; Available online: March 12, 2021

https://doi.org/10.5507/bp.2021.015

(c) 2022 The Authors; https://creativecommons.org/licenses/by/4.0/

${ }^{a}$ Department of Internal Medicine I - Cardiology, University Hospital Olomouc and Faculty of Medicine and Dentistry, Palacky University Olomouc, Czech Republic

${ }^{b}$ Cardiology Department, Institute for Clinical and Experimental Medicine, Prague, Czech Republic

'Department of Cardiology, University Hospital Pilzen, Czech Republic

${ }^{d}$ The Internal Clinic of Cardiology and Angiology of the First Faculty of Medicine and General Teaching Hospital, Czech Republic

eSt. Anne's University Hospital Brno, Czech Republic

fUniversity Hospital Brno, Czech Republic

'Liberec Hospital, Czech Republic

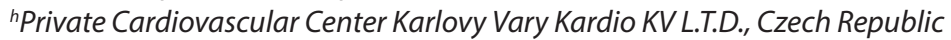

'Herzzentrum Leipzig, Germany

${ }^{j}$ Cardiological Clinic of National Institute of CV Diseases in Bratislava, Slovak Republic

kInstitute for Biostatistics and Analyses of Faculty of Medicine Brno, Czech Republic

Corresponding author: Tomas Skala, e-mail: tomasskala@gmail.com

\section{BACKGROUND}

Non-ischemic cardiomyopathy (DCM) is a common heart muscle disease characterized by an enlarged left ventricle (LV) with impaired contractility in the absence of significant coronary artery disease (CAD). It is associated with significant morbidity and mortality due to heart failure (HF) and a significant rate of sudden cardiac death (SCD). Despite recent therapeutic advances, 5-year mortality remains as high as $20 \%$ (ref. ${ }^{1}$ ). Cardiac resynchro- nization therapy (CRT) reduces morbidity and mortality in patients with symptomatic HF, reduced left ventricular ejection fraction (LVEF), and prolonged QRS duration ${ }^{2}$. Thanks to advances in heart failure pharmacotherapy, a decrease in mortality could render a benefit of ICD insufficient to justify its implantation in some patients. Moreover, the risk of ventricular arrhythmias and SCD may be sufficiently reduced with CRT-P alone due to LV reverse remodeling ${ }^{3}$. In the recent DANISH trial, implantation of a cardiac defibrillator (ICD) in patients who had 
HF that was not caused by ischemic heart disease did not provide an overall survival benefit ${ }^{4}$. Since no randomized controlled trial (RCT) has been designed to compare the effects on clinical outcomes of cardiac resynchronization therapy pacemaker (CRT-P) and cardiac resynchronization therapy with a defibrillator (CRT-D) in patients with DCM, the scientific community now wavers over the justification of an ICD implantation in this patient group 5 . The question of whether there is a benefit of CRT-D over CRT-P and at what cost is very important. ICD implantation is considerably more expensive. Especially in countries with fixed budgets for healthcare and healthcare utilization based on cost-benefit analysis, a widespread use of CRT-D devices is very limited. Moreover, the complex design of defibrillator leads can lead to a higher risk of their failure in the CRT-D systems ${ }^{6,7}$. A prospective, randomized clinical trial which would evaluate the benefit of these two approaches is greatly anticipated by the cardiologists around the world.

\section{METHODS}

\section{Study design}

The ICD-Reality study is a non-commercial, investigator-led, multicenter, prospective, randomized, blinded, controlled, interventional parallel-design trial. We aim to determine the effect of CRT-D or CRT-P implantation in DCM and HF patients.

\section{Clinical hypothesis}

Patients with symptomatic HF, with LVEF $\leq 35 \%$ and without LV mid-wall fibrosis on late gadolinium enhancement cardiovascular magnetic resonance imaging (CMR), will not benefit from CRT-D implantation compared with CRT-P only implantation.

\section{Inclusion Criteria}

$\geq 18$ years of age at the time of screening; Documented non-ischemic HF with an LVEF $\leq 35$; QRS $\geq 130 \mathrm{~ms}$; NYHA class II-IV; Signed written informed consent; NTproBNP above $200 \mathrm{pg} / \mathrm{mL}$.

\section{Exclusion Criteria}

Uncorrected congenital heart disease or valve obstruction, obstructive cardiomyopathy, active myocarditis, constrictive pericarditis, untreated hypothyroidism or hyperthyroidism, adrenal insufficiency, active vasculitis due to collagen vascular disease. Presence on the urgent waiting list for a heart transplant (UNOS category 1A or $1 \mathrm{~B}$, or equivalent). Patients on the non-urgent waiting list for a heart transplant (UNOS category 2 or 7 , or equivalent) are eligible for inclusion in the study. Recipient of any major organ transplant (e.g., lung, liver, heart). Receiving or has received cytotoxic or cytostatic chemotherapy and/or radiation therapy for treatment of a malignancy within 6 month before randomization or clinical evidence of current malignancy, with the following exceptions: basal or squamous cell carcinoma of the skin, cervical intraepithelial neoplasia, prostate cancer (if stable localized disease, with a life expectancy of $>$ 2.5 years in the opinion of the investigator). Known to be human immunodeficiency virus positive with an expected survival of less than 5 years due to HIV. Chronic kidney disease with glomerular filtration rate $<30 \mathrm{~mL} /$ min; chronic dialysis treatment. Recent (within 3 months) history of alcohol or illicit drug abuse disorder, based on self-report. Any condition (e.g., psychiatric illness) or situation that, in the investigator's opinion, could put the subject at significant risk, confound the study results, or interfere significantly with the subject's participation in the study. Unwilling to participate.

\section{Additional information related to inclusion and exclusion criteria}

The qualifying LVEF and NT-proBNP level has to be measured after a maximal tolerated pharmacotherapy of heart failure has been achieved. A non-ischemic cause of HF has to be determined by coronary angiography. Patients could be included even if they will have one or two coronary arteries with stenosis, if the extent of coronary artery disease will not be considered to be sufficient to account for the reduced LVEF. Patients with a significant coronary heart disease (CAD) will be excluded. Patients with an existing conventional pacemaker could be included if they will be willing to have the device changed or upgraded. Patients with any form of atrial fibrillation will not be excluded.

\section{The primary end-point}

Re-hospitalization for heart failure, ventricular tachycardia, major adverse cardiac events (MACE). MACE is defined as a composite of nonfatal stroke, nonfatal myocardial infarction, and cardiovascular death.

\section{The secondary end-points}

Sudden cardiac death, cardiovascular death, resuscitated cardiac arrest or sustained ventricular tachycardia, device-related complications, and change in quality of life.

\section{TIME-COURSE OF THE STUDY}

\section{Estimated number of patients needed to be screened and randomized}

600 patients are needed to be randomized according to the study power analysis. Since $35 \%$ of patients are estimated to be LG-E positive and thus excluded from randomization, 924 patients are needed to be screened.

\section{Pre-screening}

Patients with non-ischemic HF visiting an out-patient department and possibly eligible for the trial will have their pharmacotherapy optimized (Fig. 1, Table 1). A screening visit in an out-patient department will be scheduled in 1-2 months after pharmacotherapy optimization since the qualifying LVEF and NT-proBNP level has to be measured after a maximal tolerated pharmacotherapy of heart failure has been achieved. 
Table 1. The study time table.

\begin{tabular}{|c|c|c|c|c|c|c|c|c|c|c|c|c|}
\hline \multirow[b]{2}{*}{ Activity/Assessment } & \multicolumn{4}{|c|}{ Enrollment } & \multicolumn{2}{|c|}{ Allocation } & \multicolumn{5}{|c|}{ Post-allocation } & \multirow{2}{*}{\begin{tabular}{|c|} 
Close-out \\
M36 \\
\end{tabular}} \\
\hline & So - Prescreening & S1 - Screening & S2 - Screening & S3 - Screening & Randomization & MO - treatment & M6 & M12 & M18 & M24 & M30 & \\
\hline On-site visit & $x$ & $x$ & $x$ & $x$ & & $x$ & $x$ & $\mathrm{x}$ & $x$ & $x$ & $x$ & $\mathrm{x}$ \\
\hline \multicolumn{13}{|l|}{ Enrollment } \\
\hline Screening-log & $\mathrm{x}$ & $\mathrm{x}$ & $\mathrm{x}$ & $x$ & & & & & & & & \\
\hline Inclusion/exclusion criteria & & $\mathrm{x}$ & & & $x$ & & & & & & & \\
\hline \begin{tabular}{|l|} 
ICF signing \\
\end{tabular} & & $x$ & & & & & & & & & & \\
\hline \begin{tabular}{|l|} 
Randomization \\
\end{tabular} & & & & & $x$ & & & & & & & \\
\hline \multicolumn{13}{|l|}{ Intervensions } \\
\hline Pharmacotherapy optimization & $\mathrm{x}$ & & & & & & & & & & & \\
\hline CRT-D / CRT-P implantation & & & & & & $\mathrm{x}$ & & & & & & \\
\hline \multicolumn{13}{|l|}{ Assessment } \\
\hline MRI & & & $\mathrm{x}$ & & & & & & & & & \\
\hline Coronarography (optional) & & & & $x$ & & & & & & & & \\
\hline Medical history & $\mathrm{x}$ & $x$ & & & & $x$ & $x$ & $x$ & $\mathrm{x}$ & $x$ & $\mathrm{x}$ & $\mathrm{x}$ \\
\hline Vital signs / NYHA class & & $\mathrm{x}$ & & & & $\mathrm{x}$ & $\mathrm{x}$ & $\mathrm{x}$ & $\mathrm{x}$ & $\mathrm{x}$ & $\mathrm{x}$ & $\mathrm{x}$ \\
\hline Lab. tests & & $\mathrm{x}$ & & & & $\mathrm{x}$ & & $\mathrm{x}$ & & $\mathrm{x}$ & & $\mathrm{x}$ \\
\hline NT-pro-BNP & & $\mathrm{x}$ & & & & $\mathrm{x}$ & & $x$ & & $\mathrm{x}$ & & $\mathrm{x}$ \\
\hline Echocardiography & & $\mathrm{x}$ & & & & & & $\mathrm{x}$ & & $\mathrm{x}$ & & $\mathrm{x}$ \\
\hline QoL questionnaires & & $\mathrm{x}$ & & & & & & $x$ & & $x$ & & $\mathrm{x}$ \\
\hline AE/SAE & & & & & & & $\mathrm{x}$ & $x$ & $\mathrm{x}$ & $x$ & $x$ & $x$ \\
\hline
\end{tabular}

S0, Prescreening visit; S1, Screening visit; S2, Randomization visit; M0, Treatment visit; M6-M36, Follow-up visits

\section{Screening}

After the signing of informed consent, screening examination will be performed. On the consent form, participants will be asked if they agree to use of their data should they choose to withdraw from the trial. Participants will also be asked for permission for the research team to share relevant data with people from the Universities taking part in the research or from regulatory authorities, where relevant. This trial does not involve collecting biological specimens for storage. The informed consent will be obtained by attending physician in every center and a signed original will be stored in each individual center for the whole duration of the study. The screening examination will include medical history, documentation of underlying cardiac disease. The following co-morbidities will be specifically documented: peripheral arterial disease, cerebral vascular disease, pulmonary disease, diabetes mellitus, hypertension, sleep apnea, tobacco use, and any malignant disease within the last 5 years. Physical exam with vital signs, NYHA functional class, pulse rate, resting blood pressure will be performed. Cardiovascular pharmacological treatment will be documented. Standard laboratory parameters are to be recorded, including creatinine, estimated glomerular filtration rate, liver tests, TSH, NT-proBNP.

Transthoracic echocardiography will be done to confirm LVEF $\leq 35 \%$ using biplane Simpson's method. Documented will be LV end-systolic and end-diastolic diameters and volumes, thickness of ventricular septum and LV posterior wall, stroke volume, cardiac output, indexed left atrial volume, presence and grade of valvular heart disease, right ventricle diameter, TAPSE, presence and grade of pulmonary hypertension. Selective coronarography will be performed if it was not done before to exclude patients with a severe coronary heart disease. LGE-CMR imaging will be performed by an operator blinded to all other clinical data to evaluate left ventricular mid-wall fibrosis. Patients with a significant amount of fibrosis will be excluded from the study and treated according to local practice with an emphasis on ICD implantation to prevent SCD.

\section{Randomization}

After fulfilling all eligibility criteria, including maximally tolerated pharmacotherapy, subjects will be randomized by the physicians who enrolled them in a 1:1 ratio to receive CRT-D or CRT-P implantation. Randomization will be carried out by physicians enrolling patients through a stand-alone web-based CRF managed by an independent biomedicine and statistics center, independent on all randomizing personnel. The web-CRF-based allocation sequence will be based on computer-generated random numbers and will be concealed until the type of intervention is assigned.

\section{Treatment}

After randomization, devices will be implanted as soon as possible (within 2 weeks). The ICD will be programmed with anti-tachycardia pacing and shock therapy as per common praxis.

\section{Follow-up}

All patients will be followed-up for at least 3 years after the implantation. After the implantation, all patients will be examined every 6 months in an out-patient department with the assessment of medical history, vital signs, physical exam, and NT-proBNP as in the screening visit. A device control will be performed every 6 months. Patients will be evaluated for possible end-points. Deaths and hospitalizations for heart failure, stroke or arrhythmias will be recorded throughout the study duration. An Endpoint Classification Committee will adjudicate hospitalizations and deaths for causality. An independent Data Monitoring Committee will periodically review mortality data throughout the study. After device implantation the concomitant care and all possible concomitant interventions will be carried out as per common praxis. Every effort will be made to minimize lost to follow-up patients and curtail this potential bias.

\section{Quality of life}

Quality of life (QoL) is assessed at baseline and annually during follow-up. Patients will fill out the 
SF-36 Questionnaire for general QoL, the MacNew Questionnaire for disease specific QoL (ref. ${ }^{8,9}$ )

\section{Blinding}

Trial participants will be blinded to the device type for the whole duration of the trial.

The randomizing and implanting physicians will not be blinded to the type of the device. These physicians will not take care in the subsequent patients' follow-up and sign a declaration of confidentiality. The same will be true for the physicians/technicians attending to the device interrogation during follow-up visits. The device type will be hidden and physicians will sign a declaration of confidentiality. The patients' documentation will not reveal the device type. A maximum effort will be done to hide the type of the implanted device. The implantation will be intramuscular and the device interrogation followup visits will not reveal the device type. The physicians attending the follow-up visits and entering all patients' data into web-based CRF will be blinded to the implanted device type. The outcomes assessors will also be blinded to the implanted device type. In case of an emergency with a need to reveal the type of the device, i.e. lead fracture, infection in the place of implantation, ventricular tachycardia, the site principal investigator will decide to unblind the patient. This will be documented in the CRF.

\section{ADDITIONAL INFORMATION}

\section{Coordination and trial safety}

Patients will be screened in all centers and randomized via a dedicated professionally created web-based Case Report Form (CRF). CRF and randomization module creation will be done by Institute of Biostatistics and Analyses at the Faculty of Medicine of the Masaryk University (IBA MUNI Brno). All patients will be screened, included in the study, randomized, implanted and followed-up in the center where the screening will take place. All patient data will be filled in the web-based CRF by physicians in the center that enlisted the patient. All other centers will be thus blinded with respect to the patient data. For safety reasons, all patients will be followed using a daily ECG monitoring and analysis. ECG from the Home-monitoring units will be evaluated in a dedicated center. This center will evaluate the ECGs sent from the Home-monitoring units with respect to patient safety and the study end-points. If the Home-monitoring unit reveals any rhythm disturbance they will contact the patient's physician and make a record in the CRF. If a sustained ventricular tachyarrhythmia is documented on a Home-monitoring in a CRT-P patient, a decision will be made and documented about a possible crossover to CRT-D as soon as possible. Potential important protocol modifications (e.g. changes to eligibility criteria, outcomes, analyses) will be communicated with trial participants, trial registry and mentioned in the final publication. Patients who will deviate from intervention protocols (e.g. crossover to CRT-D implantation) will continue to be followed-up.

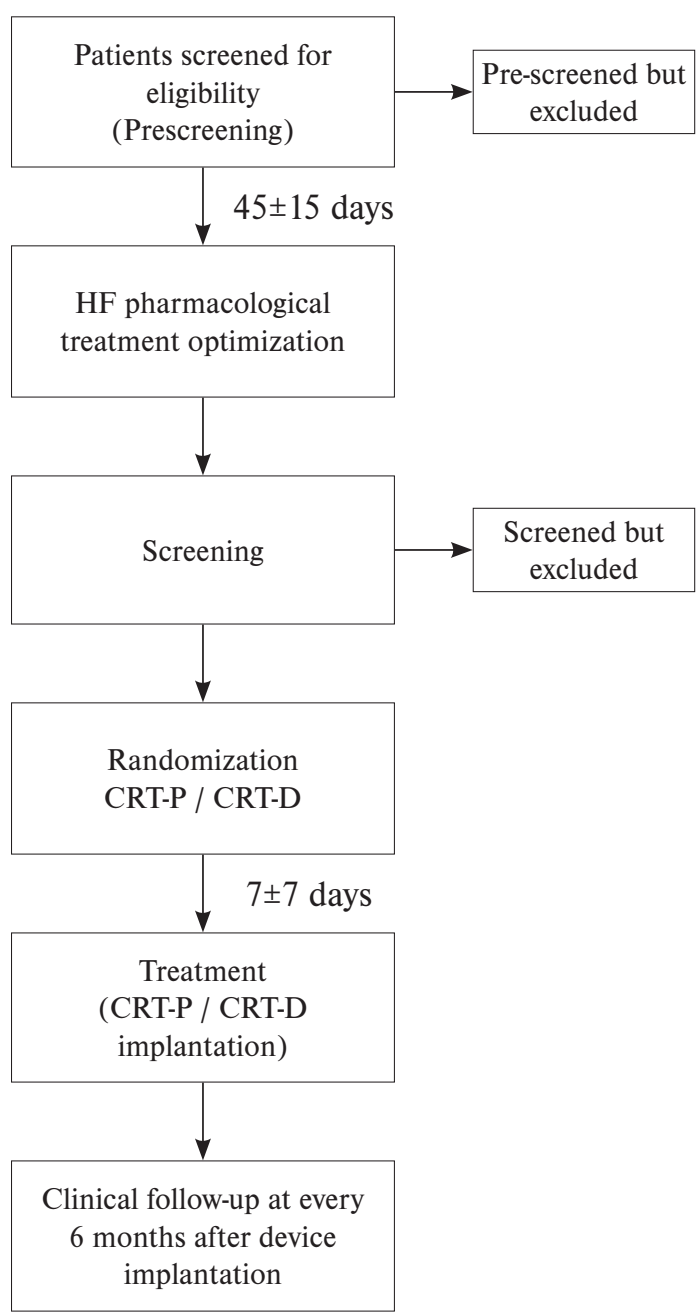

Fig. 1. The study flow-chart.

\section{DISCUSSION}

The evidence for ICDs in non-ischemic HF prior to the DANISH trial is far from being robust. There had been no randomized trials of ICD vs. no ICD in non-ischemic HF that reported reduced mortality. The most important evidence so far was a subgroup analysis of DCM patients in the SCD-HeFT trial showing a non-significant trend towards reduced mortality [hazard ratio (HR) 0.73 ; $97.5 \%$ confidence interval (CI) $0.50-1.07$ ] (ref. ${ }^{10-13}$ ). A recent meta-analysis evaluated the impact of ICD in primary prevention of SCD in DCM patients. This analysis combined the results of older RCTs and the recent DANISH trial. In contrast with the neutral results of the DANISH trial alone, the pooled analysis showed a significant reduction in total mortality in patients with ICD. The authors acknowledged that "differences in background medical therapy may confound the results of the meta-analysis" 14 .

The patients in the DANISH trial (published in 2016) were enrolled between 2008 and $2014(n=1,116)$, while patients of the previous RCTs published in the 2000s $(n=1,851)$ were enrolled between 1991 and 2002. We have witnessed a huge improvement in the treatment of HF since the older meta-analyzed studies. The rates of SCD have decreased over the last 20 year. The rate of 
death from cardiac cause, both from worsening of HF and from arrhythmias, is declining. In a recent analysis of 12 pivotal HF trials conducted from the mid-90s to 2015 $(n=40195)$, rates of sudden cardiac death had reduced by $44 \%$ over a 20 -year period ${ }^{15}$. This decline in SCD rates due to major advances in pharmacological therapy for HF is the most important reason of the DANISH trial results. In the DANISH trial, sudden death occurred in 24 patients $(4.3 \%)$ in the ICD group and in 46 patients (8.2\%) in the control group (HR 0.50; 95\% CI 0.31-0.82; $P=0.005)^{4}$. Rates of sudden death were thus very low. Only 70 patients had a sudden death over the 5 years of followup of 1116 patients. Recently, SCD rates have been further reduced by sacubitril/valsartan. Since this drug was not available during the DANISH trial, it could be argued that now it is even more difficult to reduce sudden death rates. DANISH trial was not a direct comparison of CRT-P vs CRT-D. Only $58 \%$ of patients had a CRT. And that is very important since CRT could lead to a favorable LV remodeling and thus reduce the substrate for ventricular arrhythmias.

So who should receive a CRT-P and who should receive a CRT-D? No dedicated and adequately powered trial has addressed this important question. COMPANION had a three-arm randomization to CRT-D, CRT-P and optimal medical therapy alone ${ }^{16}$. No difference was seen between CRT-D and CRT-P since this trial was not powered enough to detect it. A stand-alone trial is undoubtedly necessary. An important topic in the CRT-P/CRT-D implantation decision making process is the rate of complications. DANISH reported a not negligible complication rate. In the ICD group 3\% had a serious device infection, $2 \%$ pneumothorax and $6 \%$ received inappropriate shocks. In real-world population, complication rates are even greater ${ }^{17}$. The need for a better selection of patients is clearly perceived by clinical cardiologists. A recent survey of the European Heart Rhythm Association analyzed the changes in the indications to ICD in patients with DCM in the post-DANISH trial era. $46 \%$ of cardiologists declared to have changed their indications to ICD after the DANISH trial publication. Only 33\% declared the need of further evidence or a change in guidelines before modifying their clinical practice ${ }^{18}$.

It is crucial to exclude patients with a high SCD risk. Outside LVEF assessment, effective risk stratification with respect to SCD in DCM patients remains challenging. Ventricular fibrosis detected by LGE is a powerful predictor of ventricular tachy-arrhythmic events in DCM patients. Both the presence and extension of ventricular fibrosis correlate with the occurrence of ventricular arrhythmias and SCD, irrespective of the grade of LV dysfunction. The assessment of ventricular fibrosis has been suggested as a candidate marker to improve the decision making for ICD therapy in patients with left ventricular dysfunction ${ }^{19}$. Myocardial fibrosis promotes ventricular tachy-arrhythmias by creating a vulnerable substrate for re-entrant activity and by favoring the emergence of triggers. Currently, LGE-CMR is the reference method for the non-invasive assessment of ventricular fibrosis. Gulati, et al. tried to determine whether myocardial fibrosis de- tected by LGE-CMR is an independent predictor of mortality and SCD in DCM. After adjustment for LVEF and other conventional prognostic factors, both the presence of fibrosis and the extent were independently and incrementally associated with all-cause mortality. They have showed that the presence of a mid-wall fibrosis has a big impact on the risk profile of patients across the broad spectrum of LVEF. For example, a patient with LVEF of $35 \%$ in their cohort had a risk of death by 5 years of $12.7 \%$ (95\% CI, 6.8\%-23.0\%). When mid-wall fibrosis status was added to the risk model, the risk of death for a patient with LVEF of $35 \%$ and no mid-wall fibrosis decreased to $9.4 \%$ (95\% CI, $5.0 \%-17.5 \%$ ), while those with mid-wall fibrosis now had a predicted risk of death of $19.9 \%(95 \%$ CI, $10.8 \%-35.0 \%)\left(\right.$ ref. $\left.^{20}\right)$. Patients enlisted to our trial will thus have a CMR examination before implantation and patients with a severe fibrosis will be excluded from the study and they will receive a CRT-D.

\section{Trial ethics}

Although a stand-alone CRT-P vs. CRT-D is necessary to provide evidence of the right treatment choice, an implantation of CRT-P in a patient with a reduced LVEF can be seen as troublesome. In the proposed trial, we do our best not to include patients with a clear benefit of ICD we exclude those with CAD and to be sure these patients in high risk of SCD are not included, we will exclude patients with LV fibrosis on CMR according to a strict predefined protocol. Moreover, the whole patient group will be less risky considering SCD compared to the DANISH study population thanks to a $100 \%$ CRT implantation rate with a higher chance of a reverse LV remodeling in these non-ischemic patients in comparison with a general population due to the absence of LV scarring and thanks to the optimized and up-to-date pharmacotherapy. As mentioned above, CRT, as well as the modern pharmacotherapy, decrease mortality of these patients. Mortality of patients in our trial will be thus most probably lower than in the DANISH trial where an ICD implantation did not lead to a mortality benefit compared with patients without it.

SCD risk will never be zero in patients with HF. If we consider lowering the SCD risk of patients with DCM and HF down to zero, we will have to implant an ICD (or CRT-D) in all patients even without a solid proof of mortality benefit of ICD implantation in these patients.

Such an approach has two pitfalls.

1) Health-care expenditures: CRT-D is technologically considerably more complex than CRT-P with a roughly three times higher price. CRT-D is not even closely implanted in all patients that meet the current guidelines criteria 5 . A proof of absence of CRT-D superiority over CRT-P in a strictly defined patient population with a DCM could theoretically enable implantation of devices with a clear mortality benefit (CRT-P) in an approximately three times larger patient population in countries where health care expenditures are set according to a strict cost-benefit analysis ${ }^{7}$.

2) Complications rate: CRT-D implantation could be linked with higher complications rate compared with 
CRT-P. In an analysis of a multi-centric European cohort study (3008 patients; CRT-D, $n=1785$ ), the complications requiring surgical revision were found in 475 patients (during a mean follow-up of $41.4 \pm 29$ months) (ref. ${ }^{21}$ ). Patients with CRT-D had significantly more complications than patients with CRT-P $(\mathrm{HR}=1.68$, 95\% CI 1.27-2.23, $P=0.001)$, especially infectious complications (HR 2.10, 95\% CI 1.18-3.45, $P=0.004$ ). Moreover, this analysis did not include non-adequate ICD shocks (apart from damaged defibrillator leads). Non-adequate ICD shocks occur in $15 \%$ of patients with ICD and significantly lower the quality of their life ${ }^{22}$.

It is entirely possible that, the price of these devices, their technological complexity and the complications rate will be lowered in the future so the choice between CRT-D and CRT-P will be rendered obsolete. Nowadays, decades after the first CRT implantation, this is still far from the truth.

\section{CONCLUSION}

We present a design of a multicenter, prospective, randomized, controlled trial evaluating the benefit of implantation of CRT-D or CRT-P in non-ischemic patients with reduced LVEF and optimal pharmacotherapy without significant mid-wall myocardial fibrosis detected by CMR. If our hypothesis is confirmed, this could provide evidence for the management of these patients with a significant impact on common daily praxis and health care expenditures.

Protocol version: Version identifier: 1.0; Date: 27. 01. 2020

Trial status: not yet recruiting.

Start of recruitment: 02/2021

Approximate date when recruitment will be completed: 02/2024

Author contributions: MT, TS,RA, MF, JK, TJ, VV, AL, MV, MN, PK, RP, TR, AS, GH, ND, RH:- trial design and trial protocol approval; TS, MT: manuscript writing; MT: final approval; JJ: statistical analysis.

Conflict of interest statement interest: All authors declare no conflict of interest. All authors certify that all their affiliations with or financial involvement with any organization or entity with a financial interest in or financial conflict with the subject matter or materials discussed in the manuscript have been disclosed.

This trial is investigator-led and non-commercial.

\section{REFERENCES}

1. Felker GM, Thompson RE, Hare JM, Hruban RH, Clemetson DE, Howard DL, Baughman KL, Kasper EK. Underlying causes and long-term survival in patients with initially unexplained cardiomyopathy. N Engl J Med 2000;342(15):1077-84. doi: 10.1056/ NEJM200004133421502
2. Cleland JG, Daubert JC, Erdmann E, Freemantle N, Gras D, Kappenberger L, Tavazzi L; Cardiac Resynchronization-Heart Failure (CARE-HF) Study Investigators. The effect of cardiac resynchronization on morbidity and mortality in heart failure. N Engl J Med 2005;352(15):1539-49. doi: 10.1056/NEJMoa050496

3. Gold MR, Linde C, Abraham WT, Gardiwal A, Daubert JC. The impact of cardiac resynchronization therapy on the incidence of ventricular arrhythmias in mild heart failure. Heart Rhythm 2011;8(5):679-84. doi: 10.1016/j.hrthm.2010.12.031

4. Køber L, Thune JJ, Nielsen JC, Haarbo J, Videbæk L, Korup E, Jensen G, Hildebrandt P, Steffensen FH, Bruun NE, Eiskjær $H$, Brandes A, Thøgersen AM, Gustafsson F, Egstrup K, Videbæk R, Hassager C, Svendsen JH, Høfsten DE, Torp-Pedersen C, Pehrson S; DANISH Investigators. Defibrillator Implantation in Patients with Nonischemic Systolic Heart Failure. N Engl J Med 2016;375(13):122130. doi: 10.1056/NEJMoa1608029

5. Normand C, Linde C, Bogale N, Blomström-Lundqvist C, Auricchio A, Stellbrink C, Witte KK, Mullens W, Sticherling C, Marinskis G, Sciaraffia E, Papiashvili G, lovev S, Dickstein K. Cardiac resynchronization therapy pacemaker or cardiac resynchronization therapy defibrillator: what determines the choice?-findings from the ESC CRT Survey II. Europace 2019;21(6):918-927. doi: 10.1093/europace/euz002

6. Bryant J, Brodin H, Loveman E, Clegg A. Clinical effectiveness and cost-effectiveness of implantable cardioverter defibrillators for arrhythmias: a systematic review and economic evaluation. Int J Technol Assess Health Care 2007;23(1):63-70. doi: 10.1017/ S0266462307051586

7. Kutyifa V, Geller L, Bogyi P, Zima E, Aktas MK, Ozcan EE, Becker D, Nagy VK, Kosztin A, Szilagyi S, Merkely B. Effect of cardiac resynchronization therapy with implantable cardioverter defibrillator versus cardiac resynchronization therapy with pacemaker on mortality in heart failure patients: results of a high-volume, single-centre experience. Eur J Heart Fail 2014;16(12):1323-30. doi: 10.1002/ejhf.185

8. Ware JE Jr, Sherbourne CD. The MOS 36-item short-form health survey (SF-36). I. Conceptual framework and item selection. Med Care 1992;30(6):473-83.

9. Dixon T, Lim LL, Oldridge NB. The MacNew heart disease healthrelated quality of life instrument: reference data for users. Qual Life Res 2002;11(2):173-83. doi: 10.1023/a:1015005109731

10. Bänsch D, Antz M, Boczor S, Volkmer M, Tebbenjohanns J, Seidl K, Block M, Gietzen F, Berger J, Kuck KH. Primary prevention of sudden cardiac death in idiopathic dilated cardiomyopathy: the Cardiomyopathy Trial (CAT). Circulation 2002;105(12):1453-8. doi: 10.1161/01.cir.0000012350.99718

11. Strickberger $S A$, Hummel JD, Bartlett TG, Frumin HI, Schuger CD Beau SL, Bitar C, Morady F; AMIOVIRT Investigators. Amiodarone versus implantable cardioverter-defibrillator:randomized trial in patients with nonischemic dilated cardiomyopathy and asymptomatic nonsustained ventricular tachycardia--AMIOVIRT. J Am Coll Cardiol 2003;41(10):1707-12. doi: 10.1016/s0735-1097(03)00297-3

12. Kadish A, Dyer A, Daubert JP, Quigg R, Estes NA, Anderson KP, Calkins H, Hoch D, Goldberger J, Shalaby A, Sanders WE, Schaechter A, Levine JH; Defibrillators in Non-Ischemic Cardiomyopathy Treatment Evaluation (DEFINITE) Investigators. Prophylactic defibrillator implantation in patients with nonischemic dilated cardiomyopathy. N Engl J Med 2004;350(21):2151-8. doi: 10.1056/NEJMoa033088

13. Bardy GH, Lee KL, Mark DB, Poole JE, Packer DL, Boineau R, Domanski M, Troutman C, Anderson J, Johnson G, McNulty SE, Clapp-Channing N, Davidson-Ray LD, Fraulo ES, Fishbein DP, Luceri RM, Ip JH; Sudden Cardiac Death in Heart Failure Trial (SCD-HeFT) Investigators. Amiodarone or an implantable cardioverter-defibrillator for congestive heart failure. N Engl J Med 2005;352(3):225-37. doi: 10.1056/ NEJMoa043399

14. Stavrakis S, Asad Z, Reynolds D. Implantable Cardioverter Defibrillators for Primary Prevention of Mortality in Patients With Nonischemic Cardiomyopathy: A Meta-Analysis of Randomized Controlled Trials. J Cardiovasc Electrophysiol 2017;28(6):659-65. doi: $10.1111 /$ jce.13204

15. Shen $L$, Jhund PS, Petrie MC, Claggett BL, Barlera S, Cleland JGF, Dargie HJ, Granger CB, Kjekshus J, Køber L, Latini R, Maggioni AP, Packer M, Pitt B, Solomon SD, Swedberg K, Tavazzi L, Wikstrand J, Zannad F, Zile MR, McMurray JJV. Declining Risk of Sudden Death in Heart Failure. N Engl J Med 2017;377(1):41-51. doi: 10.1056/ NEJMoa1609758 
16. Bristow MR, Saxon LA, Boehmer J, Krueger S, Kass DA, De Marco T, Carson P, DiCarlo L, DeMets D, White BG, DeVries DW, Feldman AM; Comparison of Medical Therapy, Pacing, and Defibrillation in Heart Failure (COMPANION) Investigators. Cardiac-resynchronization therapy with or without an implantable defibrillator in advanced chronic heart failure. N Engl J Med 2004;350(21):2140-50. doi: 10.1056/NEJMoa032423

17. Hawkins NM, Grubisic M, Andrade JG, Huang F, Ding L, Gao M, Bashir J. Long-term complications, reoperations and survival following cardioverter-defibrillator implant. Heart 2018;104(3):237-43. doi: 10.1136/heartjnl-2017-311638

18. Haugaa KH, Tilz R, Boveda S, Dobreanu D, Sciaraffia E, Mansourati J, Papiashvili G, Dagres N. Implantable cardioverter defibrillator use for primary prevention in ischaemic and non-ischaemic heart diseaseindications in the post-DANISH trial era: results of the European Heart Rhythm Association survey. Europace 2017;19(4):660-64. doi: 10.1093/europace/eux089

19. Disertori $M$, Masè $M$, Ravelli F. Myocardial fibrosis predicts ventricular tachyarrhythmias. Trends Cardiovasc Med 2017;27(5):363-72. doi: 10.1016/j.tcm.2017.01.011
20. Gulati A, Jabbour A, Ismail TF, Guha K, Khwaja J, Raza S, Morarji K, Brown TD, Ismail NA, Dweck MR, Di Pietro E, Roughton M, Wage R, Daryani Y, O'Hanlon R, Sheppard MN, Alpendurada F, Lyon AR, Cook SA, Cowie MR, Assomull RG, Pennell DJ, Prasad SK. Association of fibrosis with mortality and sudden cardiac death in patients with nonischemic dilated cardiomyopathy. JAMA 2013;309(9):896-908. doi: 10.1001/jama.2013.1363

21. Barra $S$, Providência R, Boveda $S$, Duehmke R, Narayanan $K$, Chow AW, Piot O, Klug D, Defaye P, Gras D, Deharo JC, Milliez P, Da Costa A, Mondoly P, Gonzalez-Panizo J, Leclercq C, Heck P, Virdee M, Sadoul N, Le Heuzey JY, Marijon E. Device complications with addition of defibrillation to cardiac resynchronisation therapy for primary prevention. Heart 2018;104(18):1529-35. doi: 10.1136/ heartjnl-2017-312546

22. Dichtl W, Wolber T, Paoli U, Brüllmann S, Stühlinger M, Berger T, Spuller K, Strasak A, Pachinger O, Haegeli LM, Duru F, Hintringer F. Appropriate therapy but not inappropriate shocks predict survival in implantable cardioverter defibrillator patients. Clin Cardiol 2011; 34(7):433-36. doi.org/10.1002/clc.20910 